\title{
Free Term Algebras ${ }^{1}$
}

\author{
Grzegorz Bancerek \\ Faculty of Computer Science \\ Białystok Technical University \\ Wiejska 45A, 15-351 Białystok, Poland
}

\begin{abstract}
Summary. We interoduce a new characterization of algebras of normal forms of term rewriting systems [35] as algerbras of term free in itself (any function from free generators into the algebra generates endomorphism of the algebra). Introduced algebras are free in classes of algebras satisfying some sets of equalities. Their universes are subsets of all terms and the denotations of operation symbols are partially identical with the operations of construction of terms. These algebras are compiler algebras requiring some equalities of terms, e.g., associativity of addition.
\end{abstract}

MML identifier: MSAFREE4, version: $\underline{7.15 .01 \quad 4.184 .1155}$

The terminology and notation used in this paper have been introduced in the following papers: [1], [3], [13], [18], [17], [27], [15], [25], [36], [21], [22], [5], [19], [14], [41], [34], [39], [16], [11], [38], [20], [28], [29], [12], [4], [23], [37], [31], [32], [26], [42], [43], [9], [30], [33], [40], [2], [6], [7], [8], [10], and [24].

\section{Preliminaries}

In this paper $\Sigma$ is a non empty non void many sorted signature and $X$ is a non-empty many sorted set indexed by $\Sigma$.

We now state the proposition

(1) For every set $I$ and for all many sorted sets $f_{1}, f_{2}$ indexed by $I$ such that $f_{1} \subseteq f_{2}$ holds $\bigcup f_{1} \subseteq \bigcup f_{2}$.

\footnotetext{
${ }^{1}$ This work has been supported by the Polish Ministry of Science and Higher Education project "Managing a Large Repository of Computer-verified Mathematical Knowledge" (N N519 385136).

(C) 2012 University of Białystok CC-BY-SA License ver. 3.0 or later ISSN 1426-2630(p), 1898-9934(e)
} 
In the sequel $x, y$ denote sets and $i$ denotes a natural number.

Let $I$ be a set, let $X$ be a non-empty many sorted set indexed by $I$, and let $A$ be a component of $X$. We see that the element of $A$ is an element of $\bigcup X$.

Let $I$ be a set, let $X$ be a many sorted set indexed by $I$, and let $i$ be an element of $I$. Then $X(i)$ is a component of $X$.

Let $I$ be a set, let $X, Y$ be many sorted sets indexed by $I$, let $f$ be a many sorted function from $X$ into $Y$, and let $x$ be a set. Then $f(x)$ is a function from $X(x)$ into $Y(x)$.

In this article we present several logical schemes. The scheme $S c h 1$ deals with a set $\mathcal{A}$, a non-empty many sorted set $\mathcal{B}$ indexed by $\mathcal{A}$, and a binary functor $\mathcal{F}$ yielding a set, and states that:

There exists a many sorted function $f$ indexed by $\mathcal{A}$ such that for every $x$ if $x \in \mathcal{A}$, then $\operatorname{dom} f(x)=\mathcal{B}(x)$ and for every element $y$ of $\mathcal{B}(x)$ holds $f(x)(y)=\mathcal{F}(x, y)$

for all values of the parameters.

The scheme $S c h 2$ deals with a non empty set $\mathcal{A}$, non-empty many sorted sets $\mathcal{B}, \mathcal{C}$ indexed by $\mathcal{A}$, and a binary functor $\mathcal{F}$ yielding a set, and states that: There exists a many sorted function $f$ from $\mathcal{B}$ into $\mathcal{C}$ such that for every element $i$ of $\mathcal{A}$ and for every element $a$ of $\mathcal{B}(i)$ holds $f(i)(a)=\mathcal{F}(i, a)$

provided the following condition is satisfied:

- For every element $i$ of $\mathcal{A}$ and for every element $a$ of $\mathcal{B}(i)$ holds $\mathcal{F}(i, a) \in \mathcal{C}(i)$.

Let $X$ be a non empty set, let $O$ be a set, let $f$ be a function from $O$ into $X$, and let $g$ be a many sorted set indexed by $X$. Then $g \cdot f$ is a many sorted set indexed by $O$.

Let us consider $\Sigma, X$, let $F$ be a many sorted set indexed by $\Sigma$-Terms $(X)$, let $o$ be an operation symbol of $\Sigma$, and let $p$ be an argument sequence of $\operatorname{Sym}(o, X)$. One can check that $F \cdot p$ is finite sequence-like.

The following proposition is true

(2) Subtrees(the root tree of $x)=\{$ the root tree of $x\}$.

Let $f$ be a decorated tree yielding function. Observe that $\operatorname{rng} f$ is constituted of decorated trees.

The following three propositions are true:

(3) For every non empty decorated tree yielding finite sequence $p$ holds $\operatorname{Subtrees}(x$-tree $(p))=\{x$-tree $(p)\} \cup \operatorname{Subtrees}(\operatorname{rng} p)$.

(4) $\operatorname{Subtrees}(x$-tree $(\emptyset))=\{x$-tree $(\emptyset)\}$.

(5) $x$-tree $(\emptyset)=$ the root tree of $x$.

Let us observe that there exists a finite sequence which is finite-yielding, decorated tree yielding, and non empty and there exists a finite sequence which is finite-yielding, tree yielding, and non empty. 
Let $f$ be a finite-yielding function. One can check that $\operatorname{dom}_{\kappa} f(\kappa)$ is finiteyielding.

Let $p$ be a finite-yielding tree yielding finite sequence. Observe that $\overbrace{p}$ is finite.

Let $\tau$ be a finite decorated tree. Observe that $\operatorname{Subtrees}(\tau)$ is finite-membered.

Let $p$ be a finite-yielding decorated tree yielding finite sequence and let us consider $x$. Note that $x$-tree $(p)$ is finite.

One can prove the following propositions:

(6) For all finite decorated trees $\tau_{1}, \tau_{2}$ such that $\tau_{1} \in \operatorname{Subtrees}\left(\tau_{2}\right)$ holds height $\operatorname{dom} \tau_{1} \leq$ height $\operatorname{dom} \tau_{2}$.

(7) Let $p$ be a decorated tree yielding finite sequence. Suppose $p$ is finiteyielding. Let $\tau$ be a decorated tree. If $x \in \operatorname{Subtrees}(\tau)$ and $\tau \in \operatorname{rng} p$, then $x \neq y$-tree $(p)$.

Let us consider $\Sigma$ and let $X$ be a many sorted set indexed by $\Sigma$. Note that every $\Sigma$-Terms $(X)$-valued function is finite-yielding.

Next we state several propositions:

(8) For every non empty constituted of decorated trees set $X$ and for every decorated tree $\tau$ such that $\tau \in X$ holds $\operatorname{Subtrees}(\tau) \subseteq \operatorname{Subtrees}(X)$.

(9) For every non empty constituted of decorated trees set $X$ holds $X \subseteq$ $\operatorname{Subtrees}(X)$.

(10) For every term $\tau$ of $\Sigma$ over $X$ and for every $x$ such that $x \in \operatorname{Subtrees}(\tau)$ holds $x$ is a term of $\Sigma$ over $X$.

(11) For every decorated tree yielding finite sequence $p$ holds $\operatorname{rng} p \subseteq$ Subtrees $(x$-tree $(p))$.

(12) For all decorated trees $\tau_{1}, \tau_{2}$ such that $\tau_{1} \in \operatorname{Subtrees}\left(\tau_{2}\right)$ holds $\operatorname{Subtrees}\left(\tau_{1}\right) \subseteq \operatorname{Subtrees}\left(\tau_{2}\right)$.

(13) Let $X$ be a many sorted set indexed by $\Sigma$, $o$ be an operation symbol of $\Sigma$, and $p$ be a finite sequence. If $p \in \operatorname{Args}\left(o, \operatorname{Free}_{\Sigma}(X)\right)$, then $(\operatorname{Den}(o$, Free $\left.\left._{\Sigma}(X)\right)\right)(p)=\langle o$, the carrier of $\Sigma\rangle$-tree $(p)$.

Let $I$ be a set, let $A, B$ be non-empty many sorted sets indexed by $I$, and let $f$ be a many sorted function from $A$ into $B$. Observe that $\operatorname{rng}_{\kappa} f(\kappa)$ is nonempty.

Let us consider $\Sigma$. One can check that every element of $T_{\Sigma}(\mathbb{N})$ is relation-like and function-like.

Let $I$ be a set, let $A$ be a many sorted set indexed by $I$, and let $f$ be a finite sequence of elements of $I$. Observe that $A \cdot f$ is dom $f$-defined.

Let $I$ be a set, let $A$ be a many sorted set indexed by $I$, and let $f$ be a finite sequence of elements of $I$. One can verify that $A \cdot f$ is total as a dom $f$-defined binary relation.

The following propositions are true: 
(14) Let $I$ be a non empty set, $J$ be a set, and $A, B$ be many sorted sets indexed by $I$. Suppose $A \subseteq B$. Let $f$ be a function from $J$ into $I$. Then $A \cdot f \subseteq B \cdot f$ qua many sorted set indexed by $J$.

(15) Let $I$ be a set and $A, B$ be many sorted sets indexed by $I$. Suppose $A \subseteq B$. Let $f$ be a finite sequence of elements of $I$. Then $A \cdot f \subseteq B \cdot f$ qua many sorted set indexed by $\operatorname{dom} f$.

(16) For every set $I$ and for all many sorted sets $A, B$ indexed by $I$ such that $A \subseteq B$ holds $\prod A \subseteq \prod B$.

(17) Let $R$ be a weakly-normalizing binary relation with unique normal form property. If $x$ is a normal form w.r.t. $R$, then $\operatorname{nf}_{R}(x)=x$.

(18) For every weakly-normalizing binary relation $R$ with unique normal form property holds $\operatorname{nf}_{R}\left(\mathrm{nf}_{R}(x)\right)=\mathrm{nf}_{R}(x)$.

Let us consider $\Sigma, X$, let $A$ be a subset of $\operatorname{Free}(X)$, and let us consider $x$. One can verify that every element of $A(x)$ is relation-like and function-like.

Let $I$ be a set and let $A$ be a many sorted set indexed by $I$. We say that $A$ is countable if and only if:

(Def. 1) For every $x$ such that $x \in I$ holds $A(x)$ is countable.

Let $I$ be a set and let $X$ be a countable set. Note that $I \longmapsto X$ is countable as a many sorted set indexed by $I$. Note that there exists a many sorted set indexed by $I$ which is non-empty and countable.

Let $X$ be a countable many sorted set indexed by $I$, and let $x$ be a set. Note that $X(x)$ is countable.

Let $A$ be a countable set. Observe that there exists a function from $A$ into $\mathbb{N}$ which is one-to-one.

Let $I$ be a set and let $X_{0}$ be a countable many sorted set indexed by $I$. One can check that there exists a many sorted function from $X_{0}$ into $I \longmapsto \mathbb{N}$ which is " $1-1$ ".

We now state a number of propositions:

(19) Let $\Sigma$ be a set, $X$ be a many sorted set indexed by $\Sigma, Y$ be a non-empty many sorted set indexed by $\Sigma$, and $w$ be a many sorted function from $X$ into $Y$. Then $\operatorname{rng}_{\kappa} w(\kappa)$ is a many sorted subset of $Y$.

(20) Let $\Sigma$ be a set and $X$ be a countable many sorted set indexed by $\Sigma$. Then there exists a many sorted subset $Y$ of $\Sigma \longmapsto \mathbb{N}$ and there exists a many sorted function $w$ from $X$ into $\Sigma \longmapsto \mathbb{N}$ such that $w$ is "1-1" and $Y=\operatorname{rng}_{\kappa} w(\kappa)$ and for every $x$ such that $x \in \Sigma$ holds $w(x)$ is an enumeration of $X(x)$ and $Y(x)=\overline{\overline{X(x)}}$.

(21) Let $I$ be a set, $A$ be a many sorted set indexed by $I$, and $B$ be a nonempty many sorted set indexed by $I$. Then $A$ is transformable to $B$.

(22) Let $I$ be a set, $A, B, C$ be non-empty many sorted sets indexed by $I$, and $f$ be a many sorted function from $A$ into $B$. Suppose $B$ is a many 
sorted subset of $C$. Then $f$ is a many sorted function from $A$ into $C$.

(23) Let $I$ be a set and $A, B$ be many sorted sets indexed by $I$. Suppose $A$ is transformable to $B$. Let $f$ be a many sorted function from $A$ into $B$. Suppose $f$ is onto. Then there exists a many sorted function $g$ from $B$ into $A$ such that $f \circ g=\mathrm{id}_{B}$.

(24) Let $\mathfrak{A}_{1}, \mathfrak{A}_{2}$ be algebras over $\Sigma$. Suppose the algebra of $\mathfrak{A}_{1}=$ the algebra of $\mathfrak{A}_{2}$. Let $B_{1}$ be a subset of $\mathfrak{A}_{1}$ and $B_{2}$ be a subset of $\mathfrak{A}_{2}$. Suppose $B_{1}=B_{2}$. Let $o$ be an operation symbol of $\Sigma$. If $B_{1}$ is closed on $o$, then $B_{2}$ is closed on $o$.

(25) Let $\mathfrak{A}_{1}, \mathfrak{A}_{2}$ be algebras over $\Sigma$. Suppose the algebra of $\mathfrak{A}_{1}=$ the algebra of $\mathfrak{A}_{2}$. Let $B_{1}$ be a subset of $\mathfrak{A}_{1}$ and $B_{2}$ be a subset of $\mathfrak{A}_{2}$. Suppose $B_{1}=B_{2}$. Let $o$ be an operation symbol of $\Sigma$. If $B_{1}$ is closed on $o$, then $o_{B_{2}}=o_{B_{1}}$.

(26) Let $\mathfrak{A}_{1}, \mathfrak{A}_{2}$ be algebras over $\Sigma$. Suppose the algebra of $\mathfrak{A}_{1}=$ the algebra of $\mathfrak{A}_{2}$. Let $B_{1}$ be a subset of $\mathfrak{A}_{1}$ and $B_{2}$ be a subset of $\mathfrak{A}_{2}$. If $B_{1}=B_{2}$ and $B_{1}$ is operations closed, then Opers $\left(\mathfrak{A}_{2}, B_{2}\right)=\operatorname{Opers}\left(\mathfrak{A}_{1}, B_{1}\right)$.

(27) Let $\mathfrak{A}_{1}, \mathfrak{A}_{2}$ be algebras over $\Sigma$. Suppose the algebra of $\mathfrak{A}_{1}=$ the algebra of $\mathfrak{A}_{2}$. Let $B_{1}$ be a subset of $\mathfrak{A}_{1}$ and $B_{2}$ be a subset of $\mathfrak{A}_{2}$. If $B_{1}=B_{2}$ and $B_{1}$ is operations closed, then $B_{2}$ is operations closed.

(28) Let $\mathfrak{A}_{1}, \mathfrak{A}_{2}, \mathfrak{B}$ be algebras over $\Sigma$. Suppose the algebra of $\mathfrak{A}_{1}=$ the algebra of $\mathfrak{A}_{2}$. Let $\mathfrak{B}_{1}$ be a subalgebra of $\mathfrak{A}_{1}$. Suppose the algebra of $\mathfrak{B}=$ the algebra of $\mathfrak{B}_{1}$. Then $\mathfrak{B}$ is a subalgebra of $\mathfrak{A}_{2}$.

(29) For all algebras $\mathfrak{A}_{1}, \mathfrak{A}_{2}$ over $\Sigma$ such that $\mathfrak{A}_{2}$ is empty holds every many sorted function from $\mathfrak{A}_{1}$ into $\mathfrak{A}_{2}$ is a homomorphism of $\mathfrak{A}_{1}$ into $\mathfrak{A}_{2}$.

(30) Let $\mathfrak{A}_{1}, \mathfrak{A}_{2}, \mathfrak{B}_{1}$ be algebras over $\Sigma$ and $\mathfrak{B}_{2}$ be a non-empty algebra over $\Sigma$. Suppose the algebra of $\mathfrak{A}_{1}=$ the algebra of $\mathfrak{A}_{2}$ and the algebra of $\mathfrak{B}_{1}=$ the algebra of $\mathfrak{B}_{2}$. Let $h_{1}$ be a many sorted function from $\mathfrak{A}_{1}$ into $\mathfrak{B}_{1}$ and $h_{2}$ be a many sorted function from $\mathfrak{A}_{2}$ into $\mathfrak{B}_{2}$. Suppose $h_{1}=h_{2}$ and $h_{1}$ is a homomorphism of $\mathfrak{A}_{1}$ into $\mathfrak{B}_{1}$. Then $h_{2}$ is a homomorphism of $\mathfrak{A}_{2}$ into $\mathfrak{B}_{2}$.

\section{Trivial Algebras}

Let $I$ be a set and let $X$ be a many sorted set indexed by $I$. Let us observe that $X$ is trivial-yielding if and only if:

(Def. 2) For every $x$ such that $x \in I$ holds $X(x)$ is trivial.

Let $I$ be a set. Note that there exists a many sorted set indexed by $I$ which is non-empty and trivial-yielding.

Let $I$ be a set, let $\Sigma$ be a trivial-yielding many sorted set indexed by $I$, and let us consider $x$. One can check that $\Sigma(x)$ is trivial. 
Let us consider $\Sigma$ and let $\mathfrak{A}$ be an algebra over $\Sigma$. We say that $\mathfrak{A}$ is trivial if and only if:

(Def. 3) The sorts of $\mathfrak{A}$ are trivial-yielding.

Let us consider $\Sigma$. One can verify that there exists a strict algebra over $\Sigma$ which is trivial, disjoint valued, and non-empty.

Let us consider $\Sigma$ and let $\mathfrak{A}$ be a trivial algebra over $\Sigma$. One can verify that the sorts of $\mathfrak{A}$ is trivial-yielding.

Next we state four propositions:

(31) Let $\mathfrak{A}$ be a trivial non-empty algebra over $\Sigma, s$ be a sort symbol of $\Sigma$, and $e$ be an element of (the equations of $\Sigma)(s)$. Then $\mathfrak{A}=e$.

(32) For every trivial non-empty algebra $\mathfrak{A}$ over $\Sigma$ and for every set $T$ of equations of $\Sigma$ holds $\mathfrak{A}=T$.

(33) Let $\mathfrak{A}$ be a non-empty algebra over $\Sigma$ and $T$ be a non-empty trivial algebra over $\Sigma$. Then every many sorted function from $\mathfrak{A}$ into $T$ is a homomorphism of $\mathfrak{A}$ into $T$.

(34) Let $\mathfrak{T}$ be a non-empty trivial algebra over $\Sigma$ and $\mathfrak{A}$ be a non-empty subalgebra of $\mathfrak{T}$. Then the algebra of $\mathfrak{A}=$ the algebra of $\mathfrak{T}$.

\section{IMAGE}

Let us consider $\Sigma$, let $\mathfrak{A}$ be a non-empty algebra over $\Sigma$, and let $\mathfrak{C}$ be an algebra over $\Sigma$. We say that $\mathfrak{C}$ is $\mathfrak{A}$-image if and only if the condition (Def. 4) is satisfied.

(Def. 4) There exists a non-empty algebra $\mathfrak{B}$ over $\Sigma$ and there exists a many sorted function $h$ from $\mathfrak{A}$ into $\mathfrak{B}$ such that $h$ is a homomorphism of $\mathfrak{A}$ into $\mathfrak{B}$ and the algebra of $\mathfrak{C}=\operatorname{Im} h$.

Let us consider $\Sigma$ and let $\mathfrak{A}$ be a non-empty algebra over $\Sigma$. Observe that every algebra over $\Sigma$ which is $\mathfrak{A}$-image is also non-empty and there exists a non-empty strict algebra over $\Sigma$ which is $\mathfrak{A}$-image.

Let us consider $\Sigma$, let $\mathfrak{A}$ be a non-empty algebra over $\Sigma$, and let $\mathfrak{C}$ be a non-empty algebra over $\Sigma$. Let us observe that $\mathfrak{C}$ is $\mathfrak{A}$-image if and only if:

(Def. 5) There exists a many sorted function from $\mathfrak{A}$ into $\mathfrak{C}$ which is an epimorphism of $\mathfrak{A}$ onto $\mathfrak{C}$.

Let us consider $\Sigma$ and let $\mathfrak{A}$ be a non-empty algebra over $\Sigma$. An image of $\mathfrak{A}$ is an $\mathfrak{A}$-image non-empty algebra over $\Sigma$.

Let us consider $\Sigma$ and let $\mathfrak{A}$ be a non-empty algebra over $\Sigma$. Observe that there exists an image of $\mathfrak{A}$ which is disjoint valued and trivial.

One can prove the following propositions: 
(35) Let $\mathfrak{A}$ be a non-empty algebra over $\Sigma, \mathfrak{B}$ be an $\mathfrak{A}$-image algebra over $\Sigma$, $s$ be a sort symbol of $\Sigma$, and $e$ be an element of (the equations of $\Sigma)(s)$. If $\mathfrak{A}=e$, then $\mathfrak{B}=e$.

(36) Let $\mathfrak{A}$ be a non-empty algebra over $\Sigma, \mathfrak{B}$ be an $\mathfrak{A}$-image algebra over $\Sigma$, and $T$ be a set of equations of $\Sigma$. If $\mathfrak{A}=T$, then $\mathfrak{B}=T$.

\section{Term Algebras}

Let us consider $\Sigma, X$ and let $\mathfrak{A}$ be an algebra over $\Sigma$. We say that $\mathfrak{A}$ is including $\Sigma$-terms over $X$ if and only if:

(Def. 6) The sorts of $\mathfrak{A}$ are a many sorted subset of the sorts of Free $\Sigma(X)$.

Let us consider $\Sigma, X$. Note that $\operatorname{Free}_{\Sigma}(X)$ is including $\Sigma$-terms over $X$.

Let us consider $\Sigma, X$. One can check that $\operatorname{Free}_{\Sigma}(X)$ is non-empty and disjoint valued.

Let us consider $\Sigma, X$. One can check that there exists a strict algebra over $\Sigma$ which is including $\Sigma$-terms over $X$ and non-empty and there exists an algebra over $\Sigma$ which is including $\Sigma$-terms over $X$ and non-empty.

Let us consider $\Sigma, X$ and let $\mathfrak{A}$ be an including $\Sigma$-terms over $X$ algebra over $\Sigma$. We say that $\mathfrak{A}$ has all variables if and only if:

(Def. 7) FreeGenerator $(X)$ is a many sorted subset of the sorts of $\mathfrak{A}$.

We say that $\mathfrak{A}$ inherits operations if and only if the condition (Def. 8) is satisfied.

(Def. 8) Let $o$ be an operation symbol of $\Sigma$ and $p$ be a finite sequence. Suppose $p \in \operatorname{Args}\left(o, \operatorname{Free}_{\Sigma}(X)\right)$ and $\left(\operatorname{Den}\left(o, \operatorname{Free}_{\Sigma}(X)\right)\right)(p) \in$ (the sorts of $\mathfrak{A})($ the result sort of $o)$. Then $p \in \operatorname{Args}(o, \mathfrak{A})$ and $(\operatorname{Den}(o, \mathfrak{A}))(p)=(\operatorname{Den}(o$, $\left.\left.\operatorname{Free}_{\Sigma}(X)\right)\right)(p)$.

We say that $\mathfrak{A}$ is free in itself if and only if the condition (Def. 9) is satisfied.

(Def. 9) Let $f$ be a many sorted function from FreeGenerator $(X)$ into the sorts of $\mathfrak{A}$ and $G$ be a many sorted subset of the sorts of $\mathfrak{A}$. Suppose $G=$ FreeGenerator $(X)$. Then there exists a many sorted function $h$ from $\mathfrak{A}$ into $\mathfrak{A}$ such that $h$ is a homomorphism of $\mathfrak{A}$ into $\mathfrak{A}$ and $f=h \uparrow G$.

We now state two propositions:

(37) Let $\mathfrak{A}, \mathfrak{B}$ be non-empty algebras over $\Sigma$. Suppose the algebra of $\mathfrak{A}=$ the algebra of $\mathfrak{B}$. Suppose $\mathfrak{A}$ is including $\Sigma$-terms over $X$. Then $\mathfrak{B}$ is including $\Sigma$-terms over $X$.

(38) Let $\mathfrak{A}, \mathfrak{B}$ be including $\Sigma$-terms over $X$ non-empty algebras over $\Sigma$ such that the algebra of $\mathfrak{A}=$ the algebra of $\mathfrak{B}$. Then

(i) if $\mathfrak{A}$ has all variables, then $\mathfrak{B}$ has all variables,

(ii) if $\mathfrak{A}$ inherits operations, then $\mathfrak{B}$ inherits operations, and

(iii) if $\mathfrak{A}$ is free in itself, then $\mathfrak{B}$ is free in itself. 
Let $J$ be a non empty non void many sorted signature and let $\mathfrak{T}$ be a nonempty algebra over $J$. Observe that there exists a generator set of $\mathfrak{T}$ which is non-empty.

Let us consider $\Sigma, X$. Observe that $\operatorname{Free}_{\Sigma}(X)$ is free in itself, has all variables, and inherits operations.

Let us consider $\Sigma, X$. Note that every including $\Sigma$-terms over $X$ algebra over $\Sigma$ which has all variables is also non-empty and there exists an including $\Sigma$-terms over $X$ strict algebra over $\Sigma$ which is free in itself, has all variables, and inherits operations.

In the sequel $\mathfrak{A}_{0}$ denotes an including $\Sigma$-terms over $X$ non-empty algebra over $\Sigma, \mathfrak{A}_{1}$ denotes an including $\Sigma$-terms over $X$ algebra over $\Sigma$ with all variables, $\mathfrak{A}_{2}$ denotes an including $\Sigma$-terms over $X$ algebra over $\Sigma$ with all variables and inheriting operations, and $\mathfrak{A}$ denotes a free in itself including $\Sigma$-terms over $X$ algebra over $\Sigma$ with all variables and inheriting operations.

Next we state three propositions:

(39) Every element of $\mathfrak{A}_{0}$ is an element of $\operatorname{Free}_{\Sigma}(X)$ and for every sort symbol $s$ of $\Sigma$ holds every element of $\mathfrak{A}_{0}$ from $s$ is an element of $\operatorname{Free}_{\Sigma}(X)$ from $s$.

(40) Let $s$ be a sort symbol of $\Sigma$ and $x$ be an element of $X(s)$. Then the root tree of $\langle x, s\rangle$ is an element of $\mathfrak{A}_{1}$ from $s$.

(41) For every operation symbol $o$ of $\Sigma$ holds $\operatorname{Args}\left(o, \mathfrak{A}_{1}\right) \subseteq \operatorname{Args}\left(o, \operatorname{Free}_{\Sigma}(X)\right)$.

Let $\Sigma$ be a set. Observe that there exists a many sorted set indexed by $\Sigma$ which is disjoint valued and non-empty.

Let $\Sigma$ be a set and let $T$ be a disjoint valued non-empty many sorted set indexed by $\Sigma$. One can check that every many sorted subset of $T$ is disjoint valued.

Let us consider $\Sigma, X$. Observe that there exists an algebra over $\Sigma$ which is including $\Sigma$-terms over $X$ and strict.

Let us consider $\Sigma, X, \mathfrak{A}_{1}$. The canonical homomorphism of $\mathfrak{A}_{1}$ yields a many sorted function from $\operatorname{Free}_{\Sigma}(X)$ into $\mathfrak{A}_{1}$ and is defined by the conditions (Def. 10).

(Def. 10)(i) The canonical homomorphism of $\mathfrak{A}_{1}$ is a homomorphism of Free ${ }_{\Sigma}(X)$ into $\mathfrak{A}_{1}$, and

(ii) for every generator set $G$ of $\operatorname{Free}_{\Sigma}(X)$ such that $G=$ FreeGenerator $(X)$ holds $\operatorname{id}_{G}=$ (the canonical homomorphism of $\left.\mathfrak{A}_{1}\right) \uparrow G$.

Let us consider $\Sigma, X, \mathfrak{A}_{0}$. One can check that every element of $\mathfrak{A}_{0}$ is functionlike and relation-like. Let $s$ be a sort symbol of $\Sigma$. One can verify that every element of $\mathfrak{A}_{0}$ from $s$ is function-like and relation-like.

Let us consider $\Sigma, X, \mathfrak{A}_{0}$. One can verify that every element of $\mathfrak{A}_{0}$ is decorated tree-like. Let $s$ be a sort symbol of $\Sigma$. Note that every element of $\mathfrak{A}_{0}$ from $s$ is decorated tree-like.

Let us consider $\Sigma, X$. Note that every algebra over $\Sigma$ which is including $\Sigma$-terms over $X$ is also disjoint valued. 
The following propositions are true:

(42) Every element of $\mathfrak{A}_{0}$ is a term of $\Sigma$ over $X$.

(43) Let $\tau$ be an element of $\mathfrak{A}_{0}$ and $s$ be a sort symbol of $\Sigma$. If $\tau \in$ (the sorts of Free $\left.{ }_{\Sigma}(X)\right)(s)$, then $\tau \in\left(\right.$ the sorts of $\left.\mathfrak{A}_{0}\right)(s)$.

(44) For every element $\tau$ of $\mathfrak{A}_{2}$ and for every element $p$ of $\operatorname{dom} \tau$ holds $\tau\lceil p$ is an element of $\mathfrak{A}_{2}$.

(45) FreeGenerator $(X)$ is a generator set of $\mathfrak{A}_{2}$.

(46) Let $T$ be a free in itself non-empty including $\Sigma$-terms over $X$ algebra over $\Sigma, \mathfrak{A}$ be an image of $T$, and $G$ be a generator set of $T$. Suppose $G=$ FreeGenerator $(X)$. Let $f$ be a many sorted function from $G$ into the sorts of $\mathfrak{A}$. Then there exists a many sorted function $h$ from $T$ into $\mathfrak{A}$ such that $h$ is a homomorphism of $T$ into $\mathfrak{A}$ and $f=h \uparrow G$.

(47)(i) The canonical homomorphism of $\mathfrak{A}_{2}$ is an epimorphism of Free ${ }_{\Sigma}(X)$ onto $\mathfrak{A}_{2}$, and

(ii) for every sort symbol $s$ of $\Sigma$ and for every element $\tau$ of $\mathfrak{A}_{2}$ from $s$ holds (the canonical homomorphism of $\left.\mathfrak{A}_{2}\right)(s)(\tau)=\tau$.

(48) (The canonical homomorphism of $\mathfrak{A}_{2}$ ) ○ (the canonical homomorphism of $\left.\mathfrak{A}_{2}\right)=$ the canonical homomorphism of $\mathfrak{A}_{2}$.

(49) $\mathfrak{A}$ is $\operatorname{Free}_{\Sigma}(X)$-image.

\section{Satisfiability}

The following four propositions are true:

(50) Let $\mathfrak{A}$ be a non-empty algebra over $\Sigma, s$ be a sort symbol of $\Sigma$, and $\tau$ be an element of $\mathrm{T}_{\Sigma}(\mathbb{N})$ from $s$. Then $\mathfrak{A} \models \tau=\tau$.

(51) Let $A$ be a non-empty algebra over $\Sigma, s$ be a sort symbol of $\Sigma$, and $\tau_{1}$, $\tau_{2}$ be elements of $\mathrm{T}_{\Sigma}(\mathbb{N})$ from $s$. If $A \models \tau_{1}=\tau_{2}$, then $A \models \tau_{2}=\tau_{1}$.

(52) Let $\mathfrak{A}$ be a non-empty algebra over $\Sigma, s$ be a sort symbol of $\Sigma$, and $\tau_{1}$, $\tau_{2}, \tau_{3}$ be elements of $\mathrm{T}_{\Sigma}(\mathbb{N})$ from $s$. If $\mathfrak{A} \models \tau_{1}=\tau_{2}$ and $\mathfrak{A} \models \tau_{2}=\tau_{3}$, then $\mathfrak{A} \models \tau_{1}=\tau_{3}$.

(53) Let $\mathfrak{A}$ be a non-empty algebra over $\Sigma$, o be an operation symbol of $\Sigma$, and $a_{1}, a_{2}$ be finite sequences. Suppose that

(i) $\quad a_{1} \in \operatorname{Args}\left(o, \mathrm{~T}_{\Sigma}(\mathbb{N})\right)$,

(ii) $\quad a_{2} \in \operatorname{Args}\left(o, \mathrm{~T}_{\Sigma}(\mathbb{N})\right)$, and

(iii) for every natural number $i$ such that $i \in \operatorname{dom} \operatorname{Arity}(o)$ and for every sort symbol $s$ of $\Sigma$ such that $s=\operatorname{Arity}(o)(i)$ and for all elements $\tau_{1}, \tau_{2}$ of $\mathrm{T}_{\Sigma}(\mathbb{N})$ from $s$ such that $\tau_{1}=a_{1}(i)$ and $\tau_{2}=a_{2}(i)$ holds $\mathfrak{A}=\tau_{1}=\tau_{2}$.

Let $\tau_{1}, \tau_{2}$ be elements of $\mathrm{T}_{\Sigma}(\mathbb{N})$ from the result sort of $o$. Suppose $\tau_{1}=\langle o$, the carrier of $\Sigma\rangle$-tree $\left(a_{1}\right)$ and $\tau_{2}=\langle o$, the carrier of $\Sigma\rangle$-tree $\left(a_{2}\right)$. Then $\mathfrak{A} \models \tau_{1}=\tau_{2}$. 
Let us consider $\Sigma$, let $T$ be a set of equations of $\Sigma$, and let $\mathfrak{A}$ be an algebra over $\Sigma$. We say that $\mathfrak{A}$ satisfies $T$ if and only if:

(Def. 11) $\mathfrak{A} \models T$.

Let us consider $\Sigma$ and let $T$ be a set of equations of $\Sigma$. Observe that there exists an algebra over $\Sigma$ which is non-empty and trivial and satisfies $T$.

Let us consider $\Sigma$, let $T$ be a set of equations of $\Sigma$, and let $\mathfrak{A}$ be a non-empty algebra over $\Sigma$ satisfying $T$. One can verify that every non-empty algebra over $\Sigma$ which is $\mathfrak{A}$-image also satisfies $T$.

Let us consider $\Sigma$, let $\mathfrak{A}$ be an algebra over $\Sigma$, let $T$ be a set of equations of $\Sigma$, and let $G$ be a generator set of $\mathfrak{A}$. We say that $G$ is $T$-free if and only if the condition (Def. 12) is satisfied.

(Def. 12) Let $\mathfrak{B}$ be a non-empty algebra over $\Sigma$ satisfying $T$ and $f$ be a many sorted function from $G$ into the sorts of $\mathfrak{B}$. Then there exists a many sorted function $h$ from $\mathfrak{A}$ into $\mathfrak{B}$ such that $h$ is a homomorphism of $\mathfrak{A}$ into $\mathfrak{B}$ and $h \uparrow G=f$.

Let us consider $\Sigma$, let $T$ be a set of equations of $\Sigma$, and let $\mathfrak{A}$ be an algebra over $\Sigma$. We say that $\mathfrak{A}$ is $T$-free if and only if:

(Def. 13) There exists a generator set of $\mathfrak{A}$ which is $T$-free.

Let us consider $\Sigma$ and let $\mathfrak{A}$ be an algebra over $\Sigma$. The functor Equations $(\Sigma, \mathfrak{A})$ yields a set of equations of $\Sigma$ and is defined as follows:

(Def. 14) For every sort symbol $s$ of $\Sigma$ holds $(\operatorname{Equations}(\Sigma, \mathfrak{A}))(s)=\{e ; e$ ranges over elements of (the equations of $\Sigma)(s): \mathfrak{A}=e\}$.

We now state the proposition

(54) For every algebra $\mathfrak{A}$ over $\Sigma$ holds $\mathfrak{A}=\operatorname{Equations}(\Sigma, \mathfrak{A})$.

Let us consider $\Sigma$ and let $\mathfrak{A}$ be a non-empty algebra over $\Sigma$. One can verify that every $\mathfrak{A}$-image algebra over $\Sigma$ satisfies $\operatorname{Equations}(\Sigma, \mathfrak{A})$.

\section{Term Correspondence}

Now we present two schemes. The scheme TermDefEx deals with a non empty non void many sorted signature $\mathcal{A}$, a non-empty many sorted set $\mathcal{B}$ indexed by $\mathcal{A}$, a binary functor $\mathcal{F}$ yielding a set, and a binary functor $\mathcal{G}$ yielding a set, and states that:

There exists a many sorted set $F$ indexed by $\mathcal{A}$-Terms $(\mathcal{B})$ such that

(i) for every sort symbol $s$ of $\mathcal{A}$ and for every element $x$ of $\mathcal{B}(s)$ holds $F($ the root tree of $\langle x, s\rangle)=\mathcal{F}(x, s)$, and

(ii) for every operation symbol $o$ of $\mathcal{A}$ and for every argument sequence $p$ of $\operatorname{Sym}(o, \mathcal{B})$ holds $F(\operatorname{Sym}(o, \mathcal{B})$-tree $(p))=\mathcal{G}(o, F \cdot p)$ for all values of the parameters. 
The scheme TermDefUniq deals with a non empty non void many sorted signature $\mathcal{A}$, a non-empty many sorted set $\mathcal{B}$ indexed by $\mathcal{A}$, a binary functor $\mathcal{F}$ yielding a set, a binary functor $\mathcal{G}$ yielding a set, and many sorted sets $\mathcal{C}, \mathcal{D}$ indexed by $\mathcal{A}$-Terms $(\mathcal{B})$, and states that:

$$
\mathcal{C}=\mathcal{D}
$$

provided the following conditions are satisfied:

- For every sort symbol $s$ of $\mathcal{A}$ and for every element $x$ of $\mathcal{B}(s)$ holds $\mathcal{C}($ the root tree of $\langle x, s\rangle)=\mathcal{F}(x, s)$,

- For every operation symbol $o$ of $\mathcal{A}$ and for every argument sequence $p$ of $\operatorname{Sym}(o, \mathcal{B})$ holds $\mathcal{C}(\operatorname{Sym}(o, \mathcal{B})$-tree $(p))=\mathcal{G}(o, \mathcal{C} \cdot p)$,

- For every sort symbol $s$ of $\mathcal{A}$ and for every element $x$ of $\mathcal{B}(s)$ holds $\mathcal{D}($ the root tree of $\langle x, s\rangle)=\mathcal{F}(x, s)$, and

- For every operation symbol $o$ of $\mathcal{A}$ and for every argument sequence $p$ of $\operatorname{Sym}(o, \mathcal{B})$ holds $\mathcal{D}(\operatorname{Sym}(o, \mathcal{B})$-tree $(p))=\mathcal{G}(o, \mathcal{D} \cdot p)$.

Let us consider $\Sigma, X$, let $w$ be a many sorted function from $X$ into (the carrier of $\Sigma) \longmapsto \mathbb{N}$, and let $\tau$ be a function. Let us assume that $\tau$ is an element of $\operatorname{Free}_{\Sigma}(X)$. The functor $\#_{w}^{\tau}$ yields an element of $\mathrm{T}_{\Sigma}(\mathbb{N})$ and is defined by the condition (Def. 15).

(Def. 15) There exists a many sorted set $F$ indexed by $\Sigma$-Terms $(X)$ such that

(i) $\quad \#_{w}^{\tau}=F(\tau)$,

(ii) for every sort symbol $s$ of $\Sigma$ and for every element $x$ of $X(s)$ holds $F($ the root tree of $\langle x, s\rangle)=$ the root tree of $\langle w(s)(x), s\rangle$, and

(iii) for every operation symbol $o$ of $\Sigma$ and for every argument sequence $p$ of $\operatorname{Sym}(o, X)$ holds $F(\operatorname{Sym}(o, X)$-tree $(p))=\operatorname{Sym}(o,($ the carrier of $\Sigma) \longmapsto$ $\mathbb{N})$-tree $(F \cdot p)$.

We now state the proposition

(55) Let $w$ be a many sorted function from $X$ into (the carrier of $\Sigma$ ) $\longmapsto \mathbb{N}$ and $F$ be a many sorted set indexed by $\Sigma$-Terms $(X)$. Suppose that

(i) for every sort symbol $s$ of $\Sigma$ and for every element $x$ of $X(s)$ holds $F($ the root tree of $\langle x, s\rangle)=$ the root tree of $\langle w(s)(x), s\rangle$, and

(ii) for every operation symbol $o$ of $\Sigma$ and for every argument sequence $p$ of $\operatorname{Sym}(o, X)$ holds $F(\operatorname{Sym}(o, X)$-tree $(p))=\operatorname{Sym}(o,($ the carrier of $\Sigma) \longmapsto$ $\mathbb{N})$-tree $(F \cdot p)$.

Let $\tau$ be an element of $\operatorname{Free}_{\Sigma}(X)$. Then $F(\tau)=\#_{w}^{\tau}$.

Let us consider $\Sigma, X$, let $G$ be a non-empty subset of $\operatorname{Free}_{\Sigma}(X)$, and let $s$ be a sort symbol of $\Sigma$. Observe that every element of $G(s)$ is relation-like and function-like.

Next we state several propositions:

(56) Let $w$ be a many sorted function from $X$ into (the carrier of $\Sigma) \longmapsto \mathbb{N}$. Then there exists a many sorted function $h$ from $\operatorname{Free}_{\Sigma}(X)$ into $\mathrm{T}_{\Sigma}(\mathbb{N})$ such that 
(i) $\quad h$ is a homomorphism of $\operatorname{Free}_{\Sigma}(X)$ into $\mathrm{T}_{\Sigma}(\mathbb{N})$, and

(ii) for every sort symbol $s$ of $\Sigma$ and for every element $\tau$ of $\operatorname{Free}_{\Sigma}(X)$ from $s$ holds $\#_{w}^{\tau}=h(s)(\tau)$.

(57) Let $w$ be a many sorted function from $X$ into (the carrier of $\Sigma$ ) $\mathbb{N}, s$ be a sort symbol of $\Sigma$, and $x$ be an element of $X(s)$. Then $\#_{w}^{\text {the root tree of }\langle x, s\rangle}=$ the root tree of $\langle w(s)(x), s\rangle$.

(58) Let $w$ be a many sorted function from $X$ into (the carrier of $\Sigma) \longmapsto \mathbb{N}$, $o$ be an operation symbol of $\Sigma, p$ be an $\operatorname{argument}$ sequence of $\operatorname{Sym}(o, X)$, and $q$ be a finite sequence. Suppose $\operatorname{dom} q=\operatorname{dom} p$ and for every natural number $i$ and for every element $\tau$ of $\operatorname{Free}_{\Sigma}(X)$ such that $i \in \operatorname{dom} p$ and $\tau=p(i)$ holds $q(i)=\#_{w}^{\tau}$. Then $\#_{w}^{\operatorname{Sym}(o, X) \text {-tree }(p)}=\operatorname{Sym}(o$, (the carrier of $\Sigma) \longmapsto \mathbb{N})$-tree $(q)$.

(59) For every many sorted subset $Y$ of $X$ holds Free $_{\Sigma}(Y)$ is a subalgebra of $\operatorname{Free}_{\Sigma}(X)$.

(60) Let $w$ be a many sorted function from $X$ into (the carrier of $\Sigma) \longmapsto \mathbb{N}$ and $\tau$ be a term of $\Sigma$ over $X$. Then $\#_{w}^{\tau}$ is an element of Free $\Sigma\left(\operatorname{rng}_{\kappa} w(\kappa)\right)$ from the sort of $\tau$ and $\#_{w}^{\tau}$ is an element of $\mathrm{T}_{\Sigma}(\mathbb{N})$ from the sort of $\tau$.

(61) Let $w$ be a many sorted function from $X$ into (the carrier of $\Sigma$ ) $\longmapsto \mathbb{N}$ and $F$ be a many sorted set indexed by $\Sigma$-Terms $(X)$. Suppose that

(i) for every sort symbol $s$ of $\Sigma$ and for every element $x$ of $X(s)$ holds $F($ the root tree of $\langle x, s\rangle)=$ the root tree of $\langle w(s)(x), s\rangle$, and

(ii) for every operation symbol of $\Sigma$ and for every argument sequence $p$ of $\operatorname{Sym}(o, X)$ holds $F(\operatorname{Sym}(o, X)$-tree $(p))=\operatorname{Sym}(o,($ the carrier of $\Sigma) \longmapsto$ $\mathbb{N})$-tree $(F \cdot p)$.

Let $o$ be an operation symbol of $\Sigma$ and $p$ be an argument sequence of $\operatorname{Sym}(o, X)$. Then $F \cdot p \in \operatorname{Args}\left(o, \operatorname{Free}_{\Sigma}\left(\operatorname{rng}_{\kappa} w(\kappa)\right)\right)$ and $F \cdot p \in$ $\operatorname{Args}\left(o, \operatorname{Free}_{\Sigma}((\right.$ the carrier of $\left.\Sigma) \longmapsto \mathbb{N})\right)$.

(62) Let $w$ be a many sorted function from (the carrier of $\Sigma) \longmapsto \mathbb{N}$ into $X$. Then there exists a many sorted function $h$ from $T_{\Sigma}(\mathbb{N})$ into $\mathfrak{A}$ such that

(i) $\quad h$ is a homomorphism of $\mathrm{T}_{\Sigma}(\mathbb{N})$ into $\mathfrak{A}$, and

(ii) for every sort symbol $s$ of $\Sigma$ and for every natural number $i$ holds $h(s)$ (the root tree of $\langle i, s\rangle)=$ the root tree of $\langle w(s)(i), s\rangle$.

(63) Let $w$ be a many sorted function from $X$ into (the carrier of $\Sigma) \longmapsto \mathbb{N}$. Then there exists a many sorted function $h$ from FreeGenerator $(X)$ into the sorts of $\mathrm{T}_{\Sigma}(\mathbb{N})$ such that for every sort symbol $s$ of $\Sigma$ and for every element $i$ of $X(s)$ holds $h(s)$ (the root tree of $\langle i, s\rangle)=$ the root tree of $\langle w(s)(i), s\rangle$. 


\section{Free Algebras}

In the sequel $X_{0}$ is a non-empty countable many sorted set indexed by $\Sigma$ and $\mathfrak{A}_{0}$ is a free in itself including $\Sigma$-terms over $X_{0}$ algebra over $\Sigma$ with all variables and inheriting operations.

The following propositions are true:

(64) Let $h$ be a many sorted function from $T_{\Sigma}(\mathbb{N})$ into $\mathfrak{A}_{0}$. Suppose $h$ is a homomorphism of $\mathrm{T}_{\Sigma}(\mathbb{N})$ into $\mathfrak{A}_{0}$. Let $w$ be a many sorted function from $X_{0}$ into (the carrier of $\Sigma$ ) a non-empty many sorted subset $Y$ of (the carrier of $\Sigma$ ) $\longmapsto \mathbb{N}$ and there exists a subset $B$ of $\mathrm{T}_{\Sigma}(\mathbb{N})$ and there exists a many sorted function $w_{1}$ from $\operatorname{Free}_{\Sigma}(Y)$ into $\mathfrak{A}_{0}$ and there exists a many sorted function $g$ from $\mathfrak{A}_{0}$ into $\mathfrak{A}_{0}$ such that $Y=\operatorname{rng}_{\kappa} w(\kappa)$ and $B=$ the sorts of $\operatorname{Free}_{\Sigma}(Y)$ and FreeGenerator $\left(\operatorname{rng}_{\kappa} w(\kappa)\right) \subseteq B$ and $w_{1}$ is a homomorphism of $\operatorname{Free}_{\Sigma}(Y)$ into $\mathfrak{A}_{0}$ and $g$ is a homomorphism of $\mathfrak{A}_{0}$ into $\mathfrak{A}_{0}$ and $h\left\lceil B=g \circ w_{1}\right.$ and for every sort symbol $s$ of $\Sigma$ and for every natural number $i$ such that $i \in Y(s)$ there exists an element $x$ of $X_{0}(s)$ such that $w(s)(x)=i$ and $w_{1}(s)$ (the root tree of $\left.\langle i, s\rangle\right)=$ the root tree of $\langle x, s\rangle$.

(65) Let $h$ be a many sorted function from $\operatorname{Free}_{\Sigma}\left(X_{0}\right)$ into $\mathfrak{A}_{0}$. Suppose $h$ is a homomorphism of $\operatorname{Free}_{\Sigma}\left(X_{0}\right)$ into $\mathfrak{A}_{0}$. Then there exists a many sorted function $g$ from $\mathfrak{A}_{0}$ into $\mathfrak{A}_{0}$ such that $g$ is a homomorphism of $\mathfrak{A}_{0}$ into $\mathfrak{A}_{0}$ and $h=g \circ$ the canonical homomorphism of $\mathfrak{A}_{0}$.

(66) Let $o$ be an operation symbol of $\Sigma, x$ be an element of $\operatorname{Args}\left(o, \mathfrak{A}_{0}\right)$, and $x_{0}$ be an element of $\operatorname{Args}\left(o, \operatorname{Free}_{\Sigma}\left(X_{0}\right)\right)$. If $x_{0}=x$, then (the canonical homomorphism of $\left.\mathfrak{A}_{0}\right) \# x_{0}=x$.

(67) Let $o$ be an operation symbol of $\Sigma$ and $x$ be an element of $\operatorname{Args}\left(o, \mathfrak{A}_{0}\right)$. Then $\left(\operatorname{Den}\left(o, \mathfrak{A}_{0}\right)\right)(x)=\left(\right.$ the canonical homomorphism of $\left.\mathfrak{A}_{0}\right)$ (the result sort of $o)\left(\left(\operatorname{Den}\left(o, \operatorname{Free}_{\Sigma}\left(X_{0}\right)\right)\right)(x)\right)$.

(68) Let $h$ be a many sorted function from $\operatorname{Free}_{\Sigma}\left(X_{0}\right)$ into $\mathfrak{A}_{0}$. Suppose $h$ is a homomorphism of Free $\Sigma\left(X_{0}\right)$ into $\mathfrak{A}_{0}$. Let $o$ be an operation symbol of $\Sigma$ and $x$ be an element of $\operatorname{Args}\left(o, \mathfrak{A}_{0}\right)$. Then $h$ (the result sort of $\left.o\right)((\operatorname{Den}(o$, $\left.\left.\left.\mathfrak{A}_{0}\right)\right)(x)\right)=h($ the result sort of $o)\left(\left(\operatorname{Den}\left(o, \operatorname{Free}_{\Sigma}\left(X_{0}\right)\right)\right)(x)\right)$.

(69) Let $h$ be a many sorted function from Free $\Sigma\left(X_{0}\right)$ into $\mathfrak{A}_{0}$. Suppose $h$ is a homomorphism of $\operatorname{Free}_{\Sigma}\left(X_{0}\right)$ into $\mathfrak{A}_{0}$. Let $o$ be an operation symbol of $\Sigma$ and $x$ be an element of $\operatorname{Args}\left(o, \operatorname{Free}_{\Sigma}\left(X_{0}\right)\right)$. Then $h$ (the result sort of $o)\left(\left(\operatorname{Den}\left(o, \operatorname{Free}_{\Sigma}\left(X_{0}\right)\right)\right)(x)\right)=h($ the result sort of $o)((\operatorname{Den}(o$, $\left.\left.\operatorname{Free}_{\Sigma}\left(X_{0}\right)\right)\right)\left(\left(\right.\right.$ the canonical homomorphism of $\left.\left.\mathfrak{A}_{0}\right) \# x\right)$ ).

(70) Let $X_{0}, Y_{0}$ be non-empty countable many sorted sets indexed by $\Sigma, \mathfrak{A}$ be an including $\Sigma$-terms over $X_{0}$ algebra over $\Sigma$ with all variables and inheriting operations, and $h$ be a many sorted function from $\operatorname{Free}_{\Sigma}\left(Y_{0}\right)$ into $\mathfrak{A}$. Suppose $h$ is a homomorphism of $\operatorname{Free}_{\Sigma}\left(Y_{0}\right)$ into $\mathfrak{A}$. Then there 
exists a many sorted function $g$ from $\operatorname{Free}_{\Sigma}\left(Y_{0}\right)$ into $\operatorname{Free}_{\Sigma}\left(X_{0}\right)$ such that

(i) $g$ is a homomorphism of $\operatorname{Free}_{\Sigma}\left(Y_{0}\right)$ into $\operatorname{Free}_{\Sigma}\left(X_{0}\right)$,

(ii) $\quad h=($ the canonical homomorphism of $\mathfrak{A}) \circ g$, and

(iii) for every generator set $G$ of $\operatorname{Free}_{\Sigma}\left(Y_{0}\right)$ such that $G=$ FreeGenerator $\left(Y_{0}\right)$ holds $g\lceil G=h \uparrow G$.

(71) Let $w$ be a many sorted function from $X_{0}$ into (the carrier of $\Sigma$ ) $\longmapsto \mathbb{N}$, $s$ be a sort symbol of $\Sigma, \tau$ be an element of $\operatorname{Free}_{\Sigma}\left(X_{0}\right)$ from $s$, and $\tau_{1}, \tau_{2}$ be elements of $\mathrm{T}_{\Sigma}(\mathbb{N})$ from $s$. Suppose $\tau_{1}=\#_{w}^{\tau}$ and $\tau_{2}=$

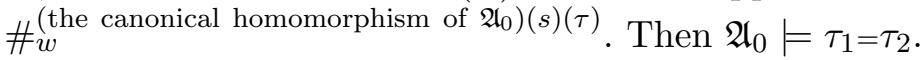

(72) Let $w$ be a many sorted function from $X_{0}$ into (the carrier of $\Sigma$ ) $\longmapsto \mathbb{N}, o$ be an operation symbol of $\Sigma, p$ be an element of $\operatorname{Args}\left(o, \operatorname{Free}_{\Sigma}\left(X_{0}\right)\right)$, and $q$ be an element of $\operatorname{Args}\left(o, \mathfrak{A}_{0}\right)$. Suppose (the canonical homomorphism of $\left.\mathfrak{A}_{0}\right) \# p=q$. Let $\tau_{1}, \tau_{2}$ be terms of $\Sigma$ over $X_{0}$. Suppose $\tau_{1}=(\operatorname{Den}(o$, Free $\left.\left._{\Sigma}\left(X_{0}\right)\right)\right)(p)$ and $\tau_{2}=\left(\operatorname{Den}\left(o, \mathfrak{A}_{0}\right)\right)(q)$. Let $\tau_{3}, \tau_{4}$ be elements of $\mathrm{T}_{\Sigma}(\mathbb{N})$ from the result sort of $o$. If $\tau_{3}=\#_{w}^{\tau_{1}}$ and $\tau_{4}=\#_{w}^{\tau_{2}}$, then $\mathfrak{A}_{0}=\tau_{3}=\tau_{4}$.

(73) Let $w$ be a many sorted function from $X_{0}$ into (the carrier of $\Sigma$ ) Suppose $w$ is "1-1". Then there exists a many sorted function $g$ from $\mathrm{T}_{\Sigma}(\mathbb{N})$ into Free ${ }_{\Sigma}\left(X_{0}\right)$ such that

(i) $\quad g$ is a homomorphism of $\mathrm{T}_{\Sigma}(\mathbb{N})$ into $\operatorname{Free}_{\Sigma}\left(X_{0}\right)$, and

(ii) for every sort symbol $s$ of $\Sigma$ and for every element $\tau$ of Free $_{\Sigma}\left(X_{0}\right)$ from $s$ holds $g(s)\left(\#_{w}^{\tau}\right)=\tau$.

(74) Let $\mathfrak{B}$ be a non-empty algebra over $\Sigma$ and $h$ be a many sorted function from Free $_{\Sigma}\left(X_{0}\right)$ into $\mathfrak{B}$. Suppose $h$ is a homomorphism of Free $\Sigma\left(X_{0}\right)$ into $\mathfrak{B}$. Let $w$ be a many sorted function from $X_{0}$ into (the carrier of $\Sigma$ ) $\longmapsto \mathbb{N}$. Suppose $w$ is "1-1". Let $s$ be a sort symbol of $\Sigma, \tau_{1}, \tau_{2}$ be elements of Free $_{\Sigma}\left(X_{0}\right)$ from $s$, and $\tau_{3}, \tau_{4}$ be elements of $\mathrm{T}_{\Sigma}(\mathbb{N})$ from $s$. If $\tau_{3}=\#_{w}^{\tau_{1}}$ and $\tau_{4}=\#_{w}^{\tau_{2}}$, then if $\mathfrak{B}=\tau_{3}=\tau_{4}$, then $h(s)\left(\tau_{1}\right)=h(s)\left(\tau_{2}\right)$.

(75) For every generator set $G$ of $\mathfrak{A}_{0}$ such that $G=$ FreeGenerator $\left(X_{0}\right)$ holds $G$ is Equations $\left(\Sigma, \mathfrak{A}_{0}\right)$-free.

$\mathfrak{A}_{0}$ is Equations $\left(\Sigma, \mathfrak{A}_{0}\right)$-free.

\section{Algebras of Normal Forms}

Let $I$ be a set, let $X, Y$ be many sorted sets indexed by $I$, let $R$ be a many sorted relation between $X$ and $Y$, and let $x$ be a set. Then $R(x)$ is a relation between $X(x)$ and $Y(x)$.

Let $I$ be a set, let $X$ be a many sorted set indexed by $I$, and let $R$ be a many sorted relation indexed by $X$. We say that $R$ is terminating if and only if:

(Def. 16) For every set $x$ such that $x \in I$ holds $R(x)$ is strongly-normalizing.

We say that $R$ has unique normal form property if and only if: 
(Def. 17) For every set $x$ such that $x \in I$ holds $R(x)$ has unique normal form property.

Let us mention that every empty set is strongly-normalizing and has unique normal form property.

One can prove the following proposition

(77) Let $I$ be a set and $A$ be a many sorted set indexed by $I$. Then there exists a many sorted relation $R$ indexed by $A$ such that $R=I \longmapsto \emptyset$ and $R$ is terminating.

Let $I$ be a set and let $X$ be a many sorted set indexed by $I$. One can verify that every many sorted relation indexed by $X$ which is empty yielding is also terminating and has unique normal form property and there exists a many sorted relation indexed by $X$ which is empty yielding.

Let $I$ be a set, let $X$ be a many sorted set indexed by $I$, let $R$ be a terminating many sorted relation indexed by $X$, and let $i$ be a set. Note that $R(i)$ is stronglynormalizing as a binary relation.

Let $R$ be a many sorted relation indexed by $X$ with unique normal form property, and let $i$ be a set. Note that $R(i)$ has unique normal form property as a binary relation.

Let us consider $\Sigma, X$ and let $R$ be a many sorted relation indexed by $\operatorname{Free}_{\Sigma}(X)$. We say that $R$ has NF-variables if and only if:

(Def. 18) For every sort symbol $s$ of $\Sigma$ holds every element of FreeGenerator $(X)(s)$ is a normal form w.r.t. $R(s)$.

We now state the proposition

(78) $x$ is a normal form w.r.t. $\emptyset$.

Let us consider $\Sigma, X$. Note that every many sorted relation indexed by Free $_{\Sigma}(X)$ which is empty yielding is also invariant and stable and has NFvariables.

Let us consider $\Sigma, X$. Observe that there exists an invariant stable many sorted relation indexed by $\operatorname{Free}_{\Sigma}(X)$ which is terminating and has NF-variables and unique normal form property.

Now we present two schemes. The scheme $A$ deals with sets $\mathcal{A}, \mathcal{B}$, a binary relation $\mathcal{C}$, and a unary predicate $\mathcal{P}$, and states that:

$\mathcal{P}[\mathcal{B}]$

provided the parameters satisfy the following conditions:

- $\mathcal{P}[\mathcal{A}]$,

- $\mathcal{C}$ reduces $\mathcal{A}$ to $\mathcal{B}$, and

- For all sets $y, z$ such that $\mathcal{C}$ reduces $\mathcal{A}$ to $y$ and $\langle y, z\rangle \in \mathcal{C}$ and $\mathcal{P}[y]$ holds $\mathcal{P}[z]$.

The scheme $B$ deals with sets $\mathcal{A}, \mathcal{B}$, a binary relation $\mathcal{C}$, and a unary predicate $\mathcal{P}$, and states that:

$\mathcal{P}[\mathcal{A}]$ 
provided the parameters meet the following requirements:

- $\mathcal{P}[\mathcal{B}]$,

- $\mathcal{C}$ reduces $\mathcal{A}$ to $\mathcal{B}$, and

- For all sets $y, z$ such that $\langle y, z\rangle \in \mathcal{C}$ and $\mathcal{P}[z]$ holds $\mathcal{P}[y]$.

Let $X$ be a non empty set, let $R$ be a strongly-normalizing binary relation on $X$ with unique normal form property, and let $x$ be an element of $X$. Then $\operatorname{nf}_{R}(x)$ is an element of $X$.

Let $I$ be a non empty set, let $X$ be a non-empty many sorted set indexed by $I$, and let $R$ be a terminating many sorted relation indexed by $X$ with unique normal form property. The functor $\operatorname{NForms}(R)$ yields a non-empty many sorted subset of $X$ and is defined as follows:

(Def. 19) For every element $i$ of $I$ holds $(\operatorname{NForms}(R))(i)=\left\{\operatorname{nf}_{R(i)}(x): x\right.$ ranges over elements of $X(i)\}$.

The scheme MSFLambda deals with a non empty set $\mathcal{A}$, a unary functor $\mathcal{F}$ yielding a non empty set, and a binary functor $\mathcal{G}$ yielding a set, and states that:

There exists a many sorted function $f$ indexed by $\mathcal{A}$ such that for every element $o$ of $\mathcal{A}$ holds

$$
\operatorname{dom} f(o)=\mathcal{F}(o) \text { and for every element } x \text { of } \mathcal{F}(o) \text { holds }
$$

$$
f(o)(x)=\mathcal{G}(o, x)
$$

for all values of the parameters.

Let us consider $\Sigma, X$ and let $R$ be a terminating invariant stable many sorted relation indexed by $\operatorname{Free}_{\Sigma}(X)$ with unique normal form property. The algebra of normal forms of $R$ yields a non-empty strict algebra over $\Sigma$ and is defined by the conditions (Def. 20).

(Def. 20)(i) The sorts of the algebra of normal forms of $R=\operatorname{NForms}(R)$, and

(ii) for every operation symbol $o$ of $\Sigma$ and for every element $a$ of $\operatorname{Args}(o$, the algebra of normal forms of $R$ ) holds (Den(o, the algebra of normal forms of $R))(a)=\mathrm{nf}_{R(\text { the result sort of } o)}\left(\left(\operatorname{Den}\left(o, \operatorname{Free}_{\Sigma}(X)\right)\right)(a)\right)$.

We now state several propositions:

(79) Let $R$ be a terminating invariant stable many sorted relation indexed by Free $_{\Sigma}(X)$ with unique normal form property and $a$ be a sort symbol of $\Sigma$. If $x \in(\operatorname{NForms}(R))(a)$, then $\operatorname{nf}_{R(a)}(x)=x$.

(80) Let $R$ be a terminating invariant stable many sorted relation indexed by $\operatorname{Free}_{\Sigma}(X)$ with unique normal form property and $g$ be a many sorted function from Free $_{\Sigma}(X)$ into Free $_{\Sigma}(X)$. Suppose $g$ is a homomorphism of Free $_{\Sigma}(X)$ into Free $_{\Sigma}(X)$. Let $s$ be a sort symbol of $\Sigma$ and $a$ be an element of $\operatorname{Free}_{\Sigma}(X)$ from $s$. Then $\operatorname{nf}_{R(s)}\left(g(s)\left(\operatorname{nf}_{R(s)}(a)\right)\right)=\operatorname{nf}_{R(s)}(g(s)(a))$.

(81) For every finite sequence $p$ holds $p_{l 0}=p$ and for every natural number $i$ such that $i \geq \operatorname{len} p$ holds $p_{l i}=\emptyset$.

(82) For all finite sequences $p, q$ holds $\left.p^{\frown}\langle x\urcorner^{\frown} q+\cdot(\operatorname{len} p+1, y)=p^{\frown}\langle y\rangle\right\urcorner q$. 
(83) For every finite sequence $p$ and for every natural number $i$ such that $i+1 \leq$ len $p$ holds $p\left\lceil(i+1)=\left(p\lceil i)^{\frown}\langle p(i+1)\rangle\right.\right.$.

(84) For every finite sequence $p$ and for every natural number $i$ such that $i+1 \leq$ len $p$ holds $p_{l i}=\langle p(i+1)\rangle^{\frown}\left(p_{l i+1}\right)$.

(85) Let $R$ be a terminating invariant stable many sorted relation indexed by $\operatorname{Free}_{\Sigma}(X)$ with unique normal form property and $g$ be a many sorted function from $\operatorname{Free}_{\Sigma}(X)$ into $\operatorname{Free}_{\Sigma}(X)$. Suppose $g$ is a homomorphism of $\operatorname{Free}_{\Sigma}(X)$ into $\operatorname{Free}_{\Sigma}(X)$. Let $h$ be a many sorted function from the algebra of normal forms of $R$ into the algebra of normal forms of $R$. Suppose that for every sort symbol $s$ of $\Sigma$ and for every element $x$ of the algebra of normal forms of $R$ from $s$ holds $h(s)(x)=\operatorname{nf}_{R(s)}(g(s)(x))$. Let $s$ be a sort symbol of $\Sigma$ and $o$ be an operation symbol of $\Sigma$. Suppose $s=$ the result sort of $o$. Let $x$ be an element of $\operatorname{Args}(o$, the algebra of normal forms of $R)$ and $y$ be an element of $\operatorname{Args}\left(o, \operatorname{Free}_{\Sigma}(X)\right)$. Suppose $x=y$. Then $\operatorname{nf}_{R(s)}((\operatorname{Den}(o$, the algebra of normal forms of $R))(h \# x))=\operatorname{nf}_{R(s)}((\operatorname{Den}(o$, $\left.\left.\left.\operatorname{Free}_{\Sigma}(X)\right)\right)(g \# y)\right)$.

Let us consider $\Sigma, X$ and let $R$ be a terminating invariant stable many sorted relation indexed by $\operatorname{Free}_{\Sigma}(X)$ with unique normal form property. One can verify that the algebra of normal forms of $R$ is including $\Sigma$-terms over $X$.

Let us consider $\Sigma, X$ and let $R$ be a terminating invariant stable many sorted relation indexed by $\operatorname{Free}_{\Sigma}(X)$ with $\mathrm{NF}$-variables and unique normal form property. Note that the algebra of normal forms of $R$ is free in itself, has all variables, and inherits operations.

\section{REFERENCES}

[1] Grzegorz Bancerek. Cardinal numbers. Formalized Mathematics, 1(2):377-382, 1990.

[2] Grzegorz Bancerek. Introduction to trees. Formalized Mathematics, 1(2):421-427, 1990.

[3] Grzegorz Bancerek. König's theorem. Formalized Mathematics, 1(3):589-593, 1990.

[4] Grzegorz Bancerek. The ordinal numbers. Formalized Mathematics, 1(1):91-96, 1990.

[5] Grzegorz Bancerek. Cartesian product of functions. Formalized Mathematics, 2(4):547$552,1991$.

[6] Grzegorz Bancerek. König's lemma. Formalized Mathematics, 2(3):397-402, 1991.

[7] Grzegorz Bancerek. Sets and functions of trees and joining operations of trees. Formalized Mathematics, 3(2):195-204, 1992.

[8] Grzegorz Bancerek. Joining of decorated trees. Formalized Mathematics, 4(1):77-82, 1993.

[9] Grzegorz Bancerek. Reduction relations. Formalized Mathematics, 5(4):469-478, 1996.

[10] Grzegorz Bancerek. Subtrees. Formalized Mathematics, 5(2):185-190, 1996.

[11] Grzegorz Bancerek. Terms over many sorted universal algebra. Formalized Mathematics, 5(2):191-198, 1996.

[12] Grzegorz Bancerek. Translations, endomorphisms, and stable equational theories. Formalized Mathematics, 5(4):553-564, 1996.

[13] Grzegorz Bancerek. Algebra of morphisms. Formalized Mathematics, 6(2):303-310, 1997.

[14] Grzegorz Bancerek. Institution of many sorted algebras. Part I: Signature reduct of an algebra. Formalized Mathematics, 6(2):279-287, 1997.

[15] Grzegorz Bancerek and Krzysztof Hryniewiecki. Segments of natural numbers and finite sequences. Formalized Mathematics, 1(1):107-114, 1990. 
[16] Grzegorz Bancerek and Artur Korniłowicz. Yet another construction of free algebra. Formalized Mathematics, 9(4):779-785, 2001.

[17] Grzegorz Bancerek and Piotr Rudnicki. On defining functions on trees. Formalized Mathematics, 4(1):91-101, 1993.

[18] Grzegorz Bancerek and Piotr Rudnicki. The set of primitive recursive functions. Formalized Mathematics, 9(4):705-720, 2001.

[19] Grzegorz Bancerek and Andrzej Trybulec. Miscellaneous facts about functions. Formalized Mathematics, 5(4):485-492, 1996.

[20] Ewa Burakowska. Subalgebras of many sorted algebra. Lattice of subalgebras. Formalized Mathematics, 5(1):47-54, 1996.

[21] Czesław Byliński. Functions and their basic properties. Formalized Mathematics, 1(1):5565, 1990.

[22] Czesław Byliński. Functions from a set to a set. Formalized Mathematics, 1(1):153-164, 1990.

[23] Czesław Byliński. Partial functions. Formalized Mathematics, 1(2):357-367, 1990.

[24] Czesław Byliński. Some basic properties of sets. Formalized Mathematics, 1(1):47-53, 1990.

[25] Agata Darmochwał. Finite sets. Formalized Mathematics, 1(1):165-167, 1990.

[26] Artur Korniłowicz. Some basic properties of many sorted sets. Formalized Mathematics, 5(3):395-399, 1996.

[27] Artur Korniłowicz. Equations in many sorted algebras. Formalized Mathematics, 6(3):363-369, 1997.

[28] Małgorzata Korolkiewicz. Homomorphisms of many sorted algebras. Formalized Mathematics, 5(1):61-65, 1996.

[29] Małgorzata Korolkiewicz. Many sorted quotient algebra. Formalized Mathematics, 5(1):79-84, 1996.

[30] Jarosław Kotowicz. Functions and finite sequences of real numbers. Formalized Mathematics, 3(2):275-278, 1992.

[31] Adam Naumowicz. On Segre's product of partial line spaces. Formalized Mathematics, 9(2):383-390, 2001.

[32] Andrzej Nędzusiak. Probability. Formalized Mathematics, 1(4):745-749, 1990.

[33] Beata Padlewska. Families of sets. Formalized Mathematics, 1(1):147-152, 1990.

[34] Beata Perkowska. Free many sorted universal algebra. Formalized Mathematics, 5(1):67$74,1996$.

[35] D.M. Gabbay S. Abramsky and T.S.E. Maibaum, editors. Handbook of Logic in Computer Science, chapter Term Rewriting Systems, pages 1-116. Oxford University Press, New York, 1992. http://www.informatik.uni-bremen.de/agbkb/lehre/rbs/texte/Klop-TR.pdf.

[36] Andrzej Trybulec. Binary operations applied to functions. Formalized Mathematics, 1(2):329-334, 1990.

[37] Andrzej Trybulec. Many sorted sets. Formalized Mathematics, 4(1):15-22, 1993.

[38] Andrzej Trybulec. Many sorted algebras. Formalized Mathematics, 5(1):37-42, 1996.

[39] Andrzej Trybulec. A scheme for extensions of homomorphisms of many sorted algebras. Formalized Mathematics, 5(2):205-209, 1996.

[40] Zinaida Trybulec. Properties of subsets. Formalized Mathematics, 1(1):67-71, 1990.

[41] Edmund Woronowicz. Many argument relations. Formalized Mathematics, 1(4):733-737, 1990.

[42] Edmund Woronowicz. Relations and their basic properties. Formalized Mathematics, 1(1):73-83, 1990.

[43] Edmund Woronowicz and Anna Zalewska. Properties of binary relations. Formalized Mathematics, 1(1):85-89, 1990.

Received May 15, 2012 